\title{
Human Interpretation of Trade-Off Diagrams in Multi-Objective Problems: Implications for Developing Interactive Decision Support Systems
}

\author{
Danielle Oprean*, Caitlin Spence**, Mark Simpson***, Randy Miller***, Saurabh Bansal ${ }^{* * *}$, Klaus Keller***, \\ Alexander Klippel*** \\ *University of Missouri, **Metropolitan Area Planning Council, ***Penn State University \\ *opreand@missouri.edu, **cspence@mapc.org, ***\{mbs278, rsm5139, sub32, kzk10, klippel\}@psu.edu
}

\begin{abstract}
The growing need for efficient and effective human decision-makers warrants a better understanding of how decision support systems (DSS) guide users to improved decisions. Decision support approaches utilize visual aids to assist decision-making, including trade-off diagrams. These visualizations help comprehension of key trade-offs among decision alternatives. However, little is known about the role of trade-off diagrams in human decision-making and the best way to present them. Here, we discuss an empirical study with two goals: 1) evaluating DSS interactivity and 2) identifying decision-making strategies with trade-off diagrams. We specifically investigate the value of interface interactivity and problem context as users make nine increasingly complex decisions. Our results suggest that problem context and interactivity separately influence ability to navigate trade-off diagrams.
\end{abstract}

\section{Introduction}

In many domains the performance of decision alternatives is evaluated on multiple objectives, which often include inherent trade-offs. For example, a policy maker may need to determine the optimal level of tax, encouraging industries to emit less carbon but curbing economic activity; a business manager may need to determine how much inventory to stock, which is costly to acquire but also provides an uncertain potential for revenue. An understanding and evaluation of these trade-offs is essential to make effective and efficient decisions. Decision support tools help to facilitate and assist these tasks. Typically, decision support tools portray trade-off information between multiple objectives in the form of trade-off diagrams. Trade-off diagrams show the impact of a change in each attribute on the overall performance of a system as well as on other attributes (Fig. 1).

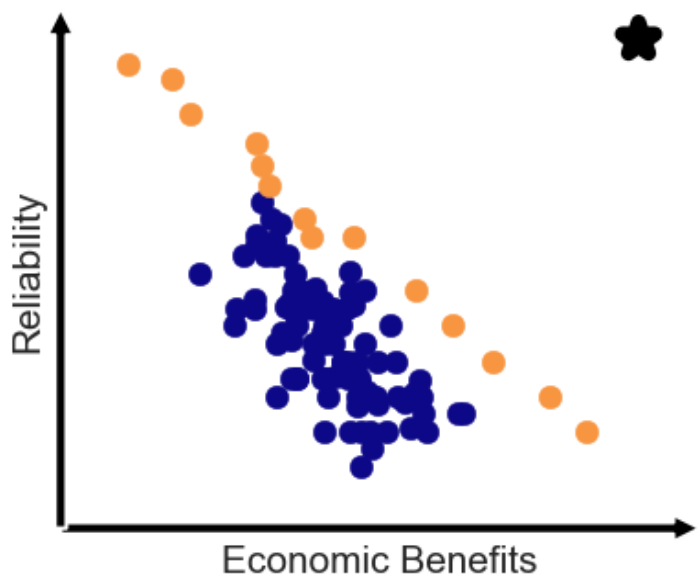

Figure 1. Example of a trade-off diagram showing alternative dominated (indigo) and non-dominated (orange) strategies. Star represents the theoretical ideal strategy.

Despite wide use of trade-off diagrams in the environmental decision support design literature [1, 2, 3 , 4], little is known about when and to what extent they are effective. Specifically, the visual format of these diagrams is meant to improve decision makers' comprehension of the problem at hand. Nevertheless, the complexity of trade-off diagrams increases with the number of objectives. As a result, trade-offs become harder to comprehend, even for experts.

Decision tools are typically contained in decision support systems (DSS), characterized as "interactive computer based systems, which help decision makers utilize data and models to solve unstructured problems" [5, p. 1]. The success of DSS is typically measured through effectiveness (decision outcomes) and efficiency (use of resources) [6]. While the design of DSS has received attention in the literature, there is scant information available for the net benefit of incorporating trade-off diagrams in DSS. Accordingly, this paper focuses on understanding the benefit of using trade-off diagrams in DSS as decision aids, when a decision maker faces a multi-attribute decision 
problem and seeks to navigate to a Pareto dominant strategy (we define these terms shortly for clarity). Here, we focus on two specific aspects of decision support tools: interactivity and contextual details, reporting on three main points on the relationship between decision-making strategy and interactive DSS using trade-off diagrams.

\section{Literature Review}

\subsection{Pareto front for multi-objective decisions}

In decision situations with multiple objectives, more than one alternative provides most-preferred performance, depending on preference among the multiple objectives. For example, policy makers are tasked with selecting energy policies to reduce carbon emissions while generating economic activity. Policy makers who value reducing carbon emissions will prefer policies reducing carbon emissions dramatically at the expense of economic activity, while policy makers valuing economic activity will favor policies that generate economic activity at the expense of reducing carbon emissions. Assuming policy makers have no motivations for selecting policies outside of reducing carbon emissions and maximizing economic productivity, no policy maker will prefer a policy with little carbon emissions reduction and little economic activity when alternatives exist with greater economic activity and higher levels of carbon emissions reduction.

Policies that can be updated to improve performance in all objectives are said to be Pareto dominated, while policies that cannot be updated to improve performance with respect to one objective without degrading performance in another objective are said to be Pareto dominant (See Fig. 1 for an illustration). The set of policies meeting Pareto Dominance criteria are on a Pareto frontier or Pareto Front [7]. The purpose of multi-objective decision support tools is to help decision makers compare candidate policies for selection in terms of multiple objectives and identify the candidate that provides the best performance given the preferences of the decision maker. Specifically, an effective DSS enables decision makers to reach the Pareto frontier and select an alternative on this frontier that maximizes net utility over multiple attributes. As will be clear in Section 3.2, our experimental design focuses on measuring the extent trade-off diagrams help decision makers reach the Pareto frontier.

\subsection{Challenges in decision approaches}

Human decision-making faces a number of challenges including characterizing the problem, weighing alternatives, unfamiliarity of problem objectives [8], trustworthiness of information [9], and overreliance on unrelated or inappropriate models for decision-making [10]. Such challenges couple with reliance on approaches based on intuition or individual values and beliefs [11].

Decision-making approaches rely on decision makers to build mental models of the problem [11]. These mental models form from numerous sources from one's beliefs and values to experiences and external stimuli [11]. Mental models present one of the key distinctions between experts and novices in decision-making, where experts draw on existing models or schema to navigate a decision while novices rely on other approaches such as intuition. The differing approaches influence the direct strategy used to make a decision. Trade-off diagrams explicitly provide relationships between various problem parameters and help decision makers develop clearer mental models, leading to better decisions.

Choosing an approach to make a decision can be influenced by the modality, format, quantity, and complexity of information. As such, decision aids and the presentation mode of information can influence decisions [12]. Budescu et al. [2014], for example, reports that decision makers using decision aids select strategies that trade off imprecision and risk rather than playing it safe. To be successful, decision aids need to present information in ways that do not bias the decision makers' understanding or choice.

\subsection{Characteristics of DSS}

Decision aids come in many forms with the general purpose of assisting with decision-making. Grouping decision aids together with other types of support forms a DSS. With a wide format of decision aids available, DSS vary in what they do and how they support decision makers [13]. Characteristics range from the user to the type of problem. Such characteristics lead to affording a user cognitively and behaviorally when making decisions.

At the cognitive level, DSS reduce mental load primarily through visualization by building on human perceptual capabilities. In other words, it is easier for a human to interpret a picture or graph than numbers alone to make sense of information. Visualization offers a visual perspective that includes users' ability to manipulate a visual representation and access a large extent of details or context about the information visualized [14]. Trade-off diagrams provide this visual representation succinctly, and are likely to improve decision makers’ performance. 
The response to the visual stimulus is often behavioral. Where visual perspectives build on users' inherent ability for visual sense-making, the response may be manipulating the representation, interacting with the system to improve the visual perspective. While DSS offer a number of ways to perform actions, extent of details or context information is highly relevant for decision-making [14]. Interactivity with a visualization system is inherently related to decisionmaking as it affords exploration of multiple perspectives of problem data.

\subsection{Interactivity}

Trade-off diagrams are commonly presented as a series of separate images breaking down multiobjective problems. DSS allow for interactive tradeoff diagrams where users manipulate not only input parameters of the diagram but also the viewing perspective. This ability to make changes to an aspect of a DSS, such as the trade-off diagram, is an affordance of the system. The affordance of interactivity enables systematic access to specific objectives in real-time, transforming, the degree of agency (direct user control) provided to a decisionmaker [15]. The effect of interactivity is behavioral, where users respond to feedback in real-time. In this study, interactivity is the ability to make changes to the trade-off diagram in real time through slider bars that manipulates the graphs, but not viewing perspective. The control for interactivity is a static trade-off diagram represented by a series of example images showing sample slider positions and the corresponding tradeoffs rather than an interactive DSS. To help isolate the effect of interactivity, we use a simplified interface to allow more control over specific affordances.

\subsection{Contextual details}

The depth and breadth of relatable information for a problem's context is part of a DSS. From a decisionmaking perspective, there is potential for one problem context to be easier to navigate than another by weighing pros and cons. Contextual details also provide more tangible situations for decisions to be made. However, this makes such systems less externally valid across disciplines. Additionally, contexts lead to preconceptions from existing mental models to influence the approach or a provided model can completely influence a decision [10]. A contextneutral option provides a simple way to determine the role of context in understanding multiple objectives in a problem. Context-neutrality provides insights into how decision makers behave without any preconceptions about the problem [12]. When faced with a realistic problem, people often incorporate their past experience or domain knowledge, biasing the results in ways impossible to tell apart from the system or problem influence. To sum, the net impact of the presence of contextual details on decision quality is not clearly established.

\section{Theory and methods}

\subsection{Hypotheses}

When faced with complex decisions, human decision makers use heuristics. These heuristics are based on mental models that decision makers construct in response to information pertaining to the problem. This information partly comes from interactivity as an engaged process where a decision maker evaluates specific alternatives and extrapolates the performance of alternatives. It may also come from contextual details enabling the decision maker to determine attributes and their interrelationships. Therefore, a stronger interactivity and contextual content in a DSS leads to better performance. Accordingly, we propose: H1: Decision makers' performance will be influenced by both interactivity and context.

Here, we focus on the interaction effect of interactivity and context. Specifically, contextual details enable decision makers to identify relevant attributes and their directional relationships that feed their mental model. Interactivity in a DSS enables decision makers to ascertain the strength of relationships between attributes. These two aspects are complementary and accordingly, we propose both have a positive impact on decision quality.

H2: A context and interactive interface will improve comprehension of system dynamics.

In the absence of context, decision makers rely on a purely mathematical understanding of a system, restricting their ability to generate a potential solution. A lack of interactivity is likely to have a similar impact. Specifically, decision makers strongly anchor their searches on solutions most salient in their recent experience. In our study, when a DSS only provides problem details in the form of specific scenarios, decision makers exhibit restricted behavior in their search for a solution.

H3: There will be a significant difference in number of common strategies based on whether the interactive or static interface is used.

\subsection{Experiment design}


We designed the experiment to test the value of trade-off diagrams for helping decision makers navigate to a Pareto dominant strategy. In this section, we describe the factorial design. In Section 3.3 we describe the interface, and in Section 3.4 we describe the problem context.

To test our hypotheses we use a 2 (interactivity) $\mathrm{x}$ 2 (context) experimental study with 4 treatments. Interactivity splits into interactive and static. Participants in the interactive condition experience real time feedback from their manipulation of the interface. The static condition provides participants with a set of images portraying examples of what the interactive interface would look like with sliders set to a range of representative combinations. Context splits into two groups based on problem framing, either context or context-neutral. The rationale is to remove undue influence of context on participants' comprehension. Differences in the context condition focus on labeling the problem: context presents an established natural resource management problem and the context-neutral condition presents the same problem without labels.
In each treatment, participants face individual versions of the problem, scaling the degree of complexity by number of objectives and levers (number of time periods controllable) through our interface. The least complex problem has a single objective and one lever while the most complex problem has three objectives and three levers. Scaling the problem into separate decisions allows for examination of the interface and decision maker performance iteratively. Figure 4 presents these 9 versions of the problem, which are also described in more detail in Section 3.4.

\subsection{Developing a decision support interface}

The support tool interface focuses primarily on trade-off diagrams. Developed through a combination of Flask ${ }^{\circ}$ and Python ${ }^{\circ}$, the web-interface allows for user input through a set of sliders. Two main layouts are used depending on the interactivity condition. The interactive condition (Fig. 2 top), provides real-time feedback in the trade-off diagram as participants alter input values through the sliders.

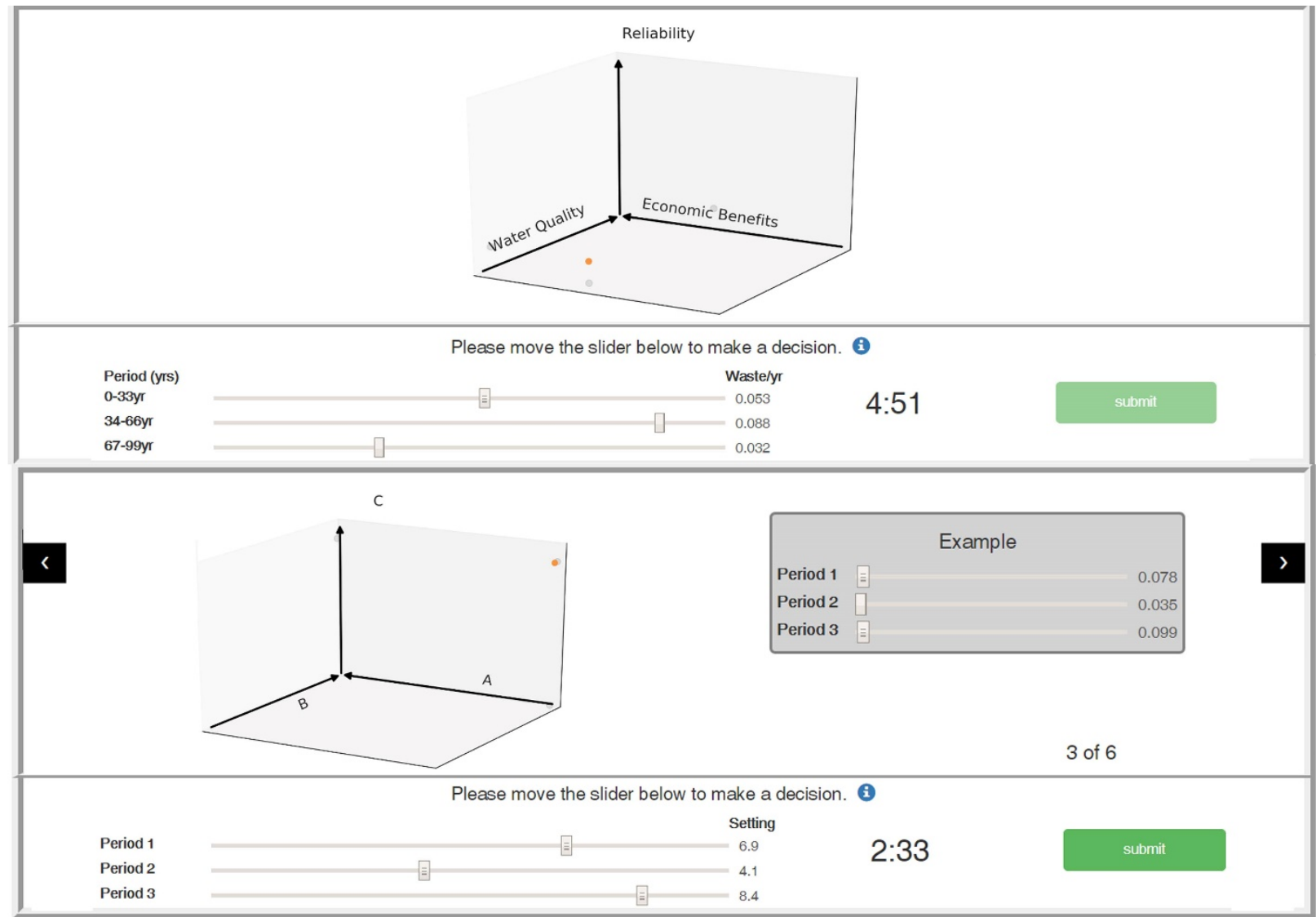

Figure 2. (Top) Screenshot of interactive interface for three objective trade-off diagram (bottom) Screenshot of static interface. 
The static condition (Fig. 2 bottom) provides six different images of example strategies and corresponding tradeoff diagrams. Users in the static condition are not able to see the trade-off diagram change in response as they adjust sliders. Each example strategy specifies different slider positions that generate specific trade-offs displayed on the diagram in each image. These strategies are selected randomly. These design features of the static and dynamic conditions are consistent with the way policy makers typically interact with DSS by looking at vignettes or sample strategies and then making decisions.

\subsection{Context description}

The problem description comes from an established natural resources management problem consisting of a lake, factory and a town $[16,1]$. The problem provides a context where decision-makers navigate multiple objectives related to the release of waste into the lake to generate economic benefits while attempting to sustain high water quality and minimize the chances of crossing an ecological tipping point into an alternate, less desirable regime (Fig. 3). The trade-off in this problem is that a high level of waste discharge increases the financial benefit, reduces water quality, and increases the chances of crossing an ecological tipping point.

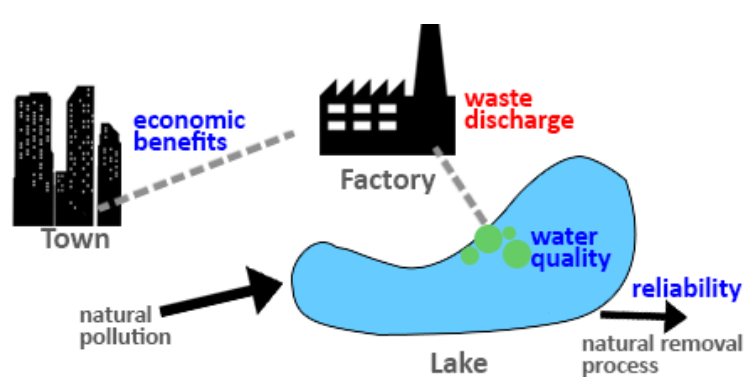

Figure. 3. The lake problem system dynamics adapted from [3] for the context condition.

A context-neutral version uses the same problem and simulation model, removing contextual labels for better comparison. Objectives are translated to letter denominated variables (economic benefit turned into A, water quality into B, and reliability into C) (Fig. 4). Note that the normative Efficient Frontiers for these 9 scenarios are available through the data and analysis in $[16,1]$. The static and dynamic performances displayed to the participants are based on these normative data. The participants are tasked with reaching as close as possible to these Efficient Frontiers. We also note the problem descriptions in the treatments are consistent with cardinal objectives and levers. The interface used in the treatments also made this feature clear.

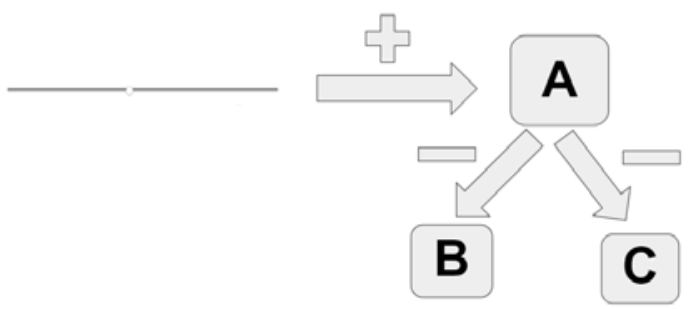

Figure 4. Context-neutral problem system dynamics with simplified labels.

Table 1. Problem format at each decision stage (context-neutral in parentheses).

\begin{tabular}{|c|c|c|}
\hline Decision & Objectives & Levers \\
\hline 1 & \multirow{3}{*}{$\begin{array}{l}\text { Maximize } \\
\text { economic } \\
\text { benefits }(\mathrm{A})\end{array}$} & $\begin{array}{c}\text { Factory waste discharge in } \\
\text { years 1-100 (Slider 1) }\end{array}$ \\
\hline 2 & & $\begin{array}{c}\text { Factory waste discharge in } \\
\text { years 1-49 (Slider 1) } \\
50-100 \text { (Slider 2) }\end{array}$ \\
\hline 3 & & $\begin{array}{c}\text { Factory waste discharge in } \\
\text { years 1-33 (Slider 1) } \\
\text { 34-66 (Slider 2) } \\
\text { 67-100 (Slider 3) }\end{array}$ \\
\hline 4 & \multirow{3}{*}{$\begin{array}{l}\text { Maximize } \\
\text { economic } \\
\text { benefits (A) } \\
\text { and water } \\
\text { quality (B) }\end{array}$} & $\begin{array}{c}\text { Factory waste discharge in } \\
\text { years 1-100 (Slider 1) }\end{array}$ \\
\hline 5 & & $\begin{array}{c}\text { Factory waste discharge in } \\
\text { years 1-49 (Slider 1) } \\
50-100 \text { (Slider } 2) \\
\end{array}$ \\
\hline 6 & & $\begin{array}{c}\text { Factory waste discharge in } \\
\text { years 1-33 (Slider 1) } \\
\text { 34-66 (Slider 2) } \\
\text { 67-100 (Slider 3) } \\
\end{array}$ \\
\hline 7 & \multirow{3}{*}{$\begin{array}{c}\text { Maximize } \\
\text { economic } \\
\text { benefits (A), } \\
\text { water quality } \\
\text { (B), and } \\
\text { reliability (C) }\end{array}$} & $\begin{array}{c}\text { Factory waste discharge in } \\
\text { years 1-100 (Slider 1) }\end{array}$ \\
\hline 8 & & $\begin{array}{c}\text { Factory waste discharge in } \\
\text { years 1-49 (Slider 1) } \\
\text { 50-100 (Slider 2) }\end{array}$ \\
\hline 9 & & $\begin{array}{c}\text { Factory waste discharge in } \\
\text { years 1-33 (Slider 1) } \\
\text { 34-66 (Slider 2) } \\
\text { 67-100 (Slider 3) }\end{array}$ \\
\hline
\end{tabular}

\subsection{Experiment protocol}


The experiment follows the protocol below. Participants enter a computer lab and occupy seats randomly. Each seat has an assigned identification number indicating which treatment a participant will complete. Entering the number into the interface opens an initial demographics survey followed by instructions about the assigned treatment through a short video tutorial.

Next, each participant provides responses to nine decision-making scenarios (Table 1). The scenarios are based on the same underlying problem, but vary by number of decision objectives in increasing order (one objective series, two objective series, and three objective series). Within each series, the decisions vary by number of levers, also in the same order (one lever, two levers, then three levers). Users have five minutes to make each decision. In order to ensure participants do not rush through the experiment, they can provide a response only after one minute per scenario.

After submitting the ninth decision, participants answer a series of questions designed to assess their decision-making process. Participants receive a cash reward based on their average set of decisions with a minimum of $\$ 7$ and a maximum up to $\$ 17$. The cash reward is based on the average percentage distance between each strategy selected by that participant and the Pareto front in the nine scenarios. A total of $\mathrm{N}=$ 142 are in the study, with an average payment of $\$ 16$.

\subsection{Measures}

To investigate both decision-making strategy and interactivity, we use several measures.

We develop a metric called "Pareto score" to quantify participants' skill in selecting a strategy near the Pareto frontier in a form comparable among different versions of the decision problem. Pareto score is calculated by comparing the Euclidean distance between a user's selected strategy and the Pareto front relative to a group of randomly generated strategies ("reference strategies"). Pareto score is the fraction of randomly generated strategies further from the Pareto front than the strategy under consideration. A score of " 1 " represents a strategy that is closer to the Pareto front than all randomly generated reference strategies, and a score of “ 0 ” represents a strategy further from the Pareto front than all of the reference strategies. This score also diagnoses if a participant's responses are likely to be randomly selected due to inattention or due to decision complexity. Because decisions made using only one lever are by definition on the Pareto front, Pareto scores are not reported for one-lever, multi-objective decisions (D4 and D7).
To understand the relative importance assigned to objectives in reaching a decision, we determine a Preference Score. Like Pareto score, the preference score is a metric normalized relative to the preferences demonstrated by a set of randomly generated "reference" strategies ranging from 0 to 1 in value. Preference score is designed to compare strategies' outcome in terms of the demonstrated values of objectives in tension in a format that is comparable among versions of the same decision problem formulated with different numbers of levers. In this problem, Economic benefits ("A") is in tension with both Water Quality (“B”) and Reliability (“C”), which are aligned. Because the Water Quality metric and Reliability metric are aligned, there is very little difference between the Economic benefits-Water Quality preference score and the Economic benefitsReliability preference score of any given strategy. In this paper, an Economic Benefits versus Water Quality objective is reported.

Lastly, six multiple-choice questions provide an assessment for whether participants understood the underlying dynamics of the lake/context-neutral problem. These questions inquire about the relationships between the levers and different objectives of the problem; correct or incorrect answers by a participant reveal understanding of the underlying relationship between each objective. These questions average into an overall comprehension score based on the number of correct responses across these questions.

\section{Analysis}

\subsection{Participants}

Participants $(\mathrm{N}=142)$ range in age from 22-44 (M $=21.63$ ) with 54 males and 88 females. The sample consists of a number of different academic backgrounds.

\subsection{Testing the first hypothesis}

We ran a two-way MANOVA with our two conditions on the two and three lever Pareto Scores, a total of six independent scores. The results indicate interactivity (Wilks $\chi=0.81, \mathrm{~F}(6,124)=4.97, \mathrm{p}=$ 0.00 , partial $\eta 2=0.19$ ), and context (Wilks $\chi=0.85$, $F(6,124)=3.75, p=0.00$, partial $\eta 2=0.15)$ affect the set of Pareto Scores individually. We find no statistically significant interaction effect between interactivity and context: Wilks $\chi=0.96, \mathrm{~F}(6,124)=$ $0.77, p=0.59$, partial $\eta 2=0.04$. These results suggest 
we partially accept $\mathrm{H} 1$ as our conditions influence Pareto Scores independently, not together.

Follow-up univariate analysis (Table 2) shows interactivity is statistically significantly correlated with Pareto Scores at a subset of decision scenarios (D2, D3, D5, and D8).

Table 2. Follow-up summary of ANOVA analysis for interactivity.

\begin{tabular}{lccccc}
\hline Decisions & $d f$ & $\mathrm{MS}$ & $\mathrm{F}$ & $\mathrm{p}$ & $\begin{array}{c}\text { partial } \\
\eta^{2}\end{array}$ \\
\hline D2 & 1 & 0.20 & 5.75 & $0.02^{*}$ & 0.04 \\
\hline D3 & 1 & 0.13 & 4.22 & $0.04 *$ & 0.03 \\
\hline D5 & 1 & 1.20 & 16.31 & $0.00^{* *}$ & 0.11 \\
\hline D8 & 1 & 0.68 & 11.57 & $0.00 * *$ & 0.08 \\
\hline Error & 129 & & & & \\
\hline
\end{tabular}

Note. - * ${ }^{*}<0.05 ; * * \mathrm{p}<0.001$

Pareto scores within the interactive condition are statistically significantly higher than scores in the static condition except in the three-objective decision 8 (D8) (Figure 4, p < 0.05).

Table 3. Mean differences on Pareto Score by interactivity condition.

\begin{tabular}{llll}
\hline \multirow{2}{*}{ Decisions } & Condition & $\mathrm{M}$ & Std. Error \\
\hline & interactive & 0.89 & 0.02 \\
\cline { 2 - 4 } & static & 0.81 & 0.02 \\
\hline \multirow{2}{*}{ D3 } & interactive & 0.91 & 0.02 \\
\cline { 2 - 4 } & static & 0.84 & 0.02 \\
\hline D5 & interactive & 0.74 & 0.03 \\
\cline { 2 - 4 } & static & 0.55 & 0.03 \\
\hline \multirow{2}{*}{ D8 } & interactive & 0.43 & 0.03 \\
\cline { 2 - 4 } & static & 0.57 & 0.04 \\
\hline
\end{tabular}

Follow-up univariate analysis (Table 4) reveals statistically significant relationships between context and Pareto Scores at a subset of decision scenarios (D2, D3). There is a near significance for D8.

Table 4. Follow-up summary of ANOVA analysis for context.

\begin{tabular}{lccccc}
\hline Decisions & $d f$ & $\mathrm{MS}$ & $\mathrm{F}$ & $\mathrm{p}$ & $\begin{array}{c}\text { partial } \\
\eta^{2}\end{array}$ \\
\hline $\mathrm{D} 2$ & 1 & 0.66 & 19.01 & $0.00^{* *}$ & 0.13 \\
\hline $\mathrm{D} 3$ & 1 & 0.37 & 12.07 & $0.00^{* *}$ & 0.09 \\
\hline $\mathrm{D} 8$ & 1 & 0.21 & 3.60 & 0.06 & 0.03 \\
\hline Error & 129 & & & & \\
\hline Note. $-* \mathrm{p}<0.05 ; * * \mathrm{p}<0.001$ & &
\end{tabular}

In both significant cases, Pareto scores are generally higher in the context-neutral condition than the lake condition (Table 5). In the near significant case, strategies in the context-neutral condition ( $\mathrm{M}=$ $0.54, \mathrm{SE}=0.03$ ) also outperformed the lake condition $(\mathrm{M}=0.46, \mathrm{SE}=0.03)$.

Table 5. Mean differences on Pareto Score by context condition.

\begin{tabular}{llll}
\hline Decisions & Condition & $\mathrm{M}$ & Std. Error \\
\hline \multirow{2}{*}{ D2 } & interactive & 0.78 & 0.02 \\
\cline { 2 - 4 } & static & 0.92 & 0.02 \\
\hline \multirow{2}{*}{ D3 } & interactive & 0.82 & 0.02 \\
\cline { 2 - 4 } & static & 0.93 & 0.02 \\
\hline
\end{tabular}

\subsection{Testing the second hypothesis}

We use a two-way ANOVA with the two conditions to assess variation in comprehension score between and among groups. There is no significant interaction effect between interaction and context on system dynamics comprehension: $\mathrm{F}(1,135)=0.31$, $\mathrm{p}$ $=0.58$, partial $\eta 2=0.00$. Context is the most important factor explaining comprehension of system dynamics: $F(1,135)=120.80, p=0.00$, partial $\eta 2=047$. The 
lake context $(\mathrm{M}=0.59 \mathrm{SE}=0.02)$ improves comprehension better than context-neutral $(\mathrm{M}=0.28$ $\mathrm{SE}=0.02)$. Based on this finding, we reject $\mathrm{H} 2$ as interactivity has no significant influence on comprehension of system dynamics.

\subsection{Testing the third hypothesis}

The type of strategy selected by each group is measured through preference score. KolmogorovSmirnov tests compare the distributions of preference scores at each decision stage and each betweensubjects context group to the corresponding group in the other interactivity condition. For example, the preference score selected by the context-neutral interactive group is compared with the preference scores of strategies by the context-neutral static group. All pairwise comparisons between groups at the twoobjective decision stages (decisions 4, 5, and 6) indicate statistically significant distributions of preference score $(\mathrm{p}<0.01)$ between interactive and static conditions (Fig. 5).

Statistically significant preference score difference also exists in the context group at decision 2 ( $p<0.01)$, the context group at decision $3(\mathrm{p}<0.05)$, and the context group at decisions 7 and $8(\mathrm{p}<0.01)$. Two-objective strategies selected by the interactive groups favor the Water Quality/B objective more often than strategies selected by static groups (Fig. 5). Based on these findings, we partially accept H3s.

\section{Preferences displayed in two-objective strategies}

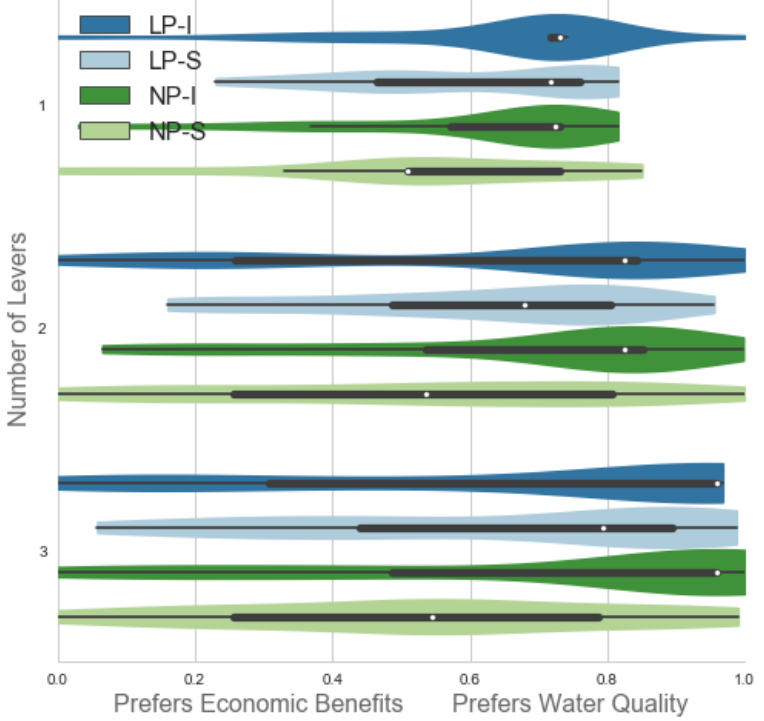

Figure 5. Comparison of preference scores demonstrated through selection of two-

\author{
objective decisions. LP-I: lake problem, \\ interactive condition. LP-S: lake problem, \\ static condition. NP-I: neutral problem, \\ interactive condition. NP-S: neutral problem, \\ static condition.
}

\section{Discussion}

While it seems intuitive that users in the interactive condition would perform better, as they could more fully explore the dynamics of the system through the trade-off diagrams, this was not always the case. We speculate that participants in the static condition are forced to create a more complete mental model of the system in order to formulate an approach, since they could not employ a strategy of manipulating the levers more or less randomly until they found a satisfactory decision. Alternatively, participants in the static condition may have chosen to emulate one of the provided examples if they are able to recognize that a particular example strategy is high quality. This approach may have created a more straightforward pathway toward the Pareto front than interactive manipulation.

Interestingly, decision effectiveness is not related to any joint influence from context or interactivity. This supports the notion that context pertains to the cognitive affordance of the visualized trade-off diagrams while interactivity is the behavioral component. This finding also suggests that underlying mental models of the trade-off diagrams are not strengthened through a combination of afforded interaction and relatable context. Rather, decision effectiveness is based on either interactivity or context individually, a departure from the idea that both aspects make up the visual perspective [14]. In the most complex scenario we found no statistically significant relationship between performance and either condition, suggesting there may a threshold in decision complexity beyond which trade-off diagrams alone are no longer sufficient to assist decisionmaking.

However, the hypothesis that having a context would enable a better understanding of the problem is supported, as participants in the context condition show a clearer understanding of system dynamics.

Lastly, the hypothesis the number of common strategies will differ between interactive and static conditions is supported though the difference was not statistically significant at all decision stages. The difference is demonstrated most clearly by comparing preference scores at the two-objective decision scenarios, where most members of the interactive group chose similar strategies while the static 
condition chose strategies that demonstrated more widely dispersed preferences. This may indicate that participants in the interactive condition are motivated by the threshold in the system dynamics, which they are able to identify by experimentally adjusting the levers. This threshold is not evident in the singleobjective version of the problem, and would have been evident but potentially more difficult to navigate in the three-objective version of the problem. Participants in the static conditions might not have recognized the threshold in the second-objective performance, or may have based their strategies on the examples given which span a range of preferences. This suggests the particular system dynamics native to a decision problem may influence users' interactivity-driven decision-making strategy.

\section{Implications}

Our findings have implications for the design of efficient DSS using trade-off diagrams. First, contextual information in the trade-off diagrams significantly increases decision quality. In many situations contextual knowledge may be complex and or difficult to express. Our findings suggest the benefit of providing these details is likely to be worth the effort. Interactivity also improves decision quality, albeit to a lesser extent. Second, the lack of evidence for interactive influence of interactivity and context on decision quality implies these two features of a DSS are substitutes. In light of the first implication, this substitutability implies that in the presence of limited resources, it may be prudent to first add context to the decision-diagrams, and then, resources permitting, one should add other features such as interactivity. Finally, the benefit of trade-off diagrams decreases with problem complexity. As a result no one-catch-all approach can be used to guide the design of DSS. For complex and large scale decision problems, careful studies for the efficiency of specific support tools, either individually or simultaneously, are warranted.

\section{Conclusion and outlook}

In domains where decisions are based on a number of objectives, decision-makers need to be able to understand and address trade-offs. DSS assist decision-makers through various decision aids and formats of presenting information. These characteristics lead to affordances that can impact the mental models of decision makers. Specifically, these characteristics influence not only the approach to reach a decision but the actual strategy used to reach a specific outcome.
To examine the effectiveness of DSS for assisting decision makers in multi-objective problems, we pose a larger question asking how affordances of DSS can help decision-makers navigate to a Pareto-dominant strategy. We focus our study on one common visual method, trade-off diagrams, to distill its role in assisting with decision-making. We identify a cognitive (context) and a behavioral (interactivity) aspect of visualization to identify the effectiveness of trade-off diagrams in a problem that become increasingly complex. We find that trade-off diagrams alone are sufficient for problems with fewer objectives. However, as problem complexity increases, trade-off diagrams no longer support decision-making effectively.

This pilot study provides the opportunity to not only explore trade-off diagrams further, but also to examine the role of other visualization methods used in a DSS. Limitations of the study include the limited interaction capabilities of our DSS meant to provide a focused look at a specific aspect found in most DSS. Follow-ups to this study will further investigate the role of context and interactivity in the formation of mental models that lead to decision-maker approaches through more complex DSS interfaces.

\section{Acknowledgments}

We would like to acknowledge the co-support of the Penn State Center for Climate Risk Management and ChoroPhronesis. The experiments reported in the paper were conducted at the Laboratory for Economics, Management, and Auctions (LEMA) at Smeal College of Business.

\section{References}

[1] Singh, Riddhi; Reed, Patrick M.; Keller, Klaus (2015): Many-objective robust decision making for managing an ecosystem with a deeply uncertain threshold response. In E\&S 20 (3), pp. 1-32. DOI: 10.5751/ES-07687-200312.

[2] Kasprzyk, Joseph R.; Nataraj, Shanthi; Reed, Patrick M.; Lempert, Robert J. (2013): Many objective robust decision making for complex environmental systems undergoing change. In Environmental Modelling \& Software 42, pp. 5571. DOI: 10.1016/j.envsoft.2012.12.007.

[3] Hadka, David; Herman, Jonathan; Reed, Patrick; Keller, Klaus (2015): An open source framework for manyobjective robust decision making. In Environmental Modelling \& Software 74, pp. 114-129. DOI: 10.1016/j.envsoft.2015.07.014.

[4] Garner, Gregory; Reed, Patrick; Keller, Klaus (2016): Climate risk management requires explicit representation of 
societal trade-offs. In Climatic Change 134 (4), pp. 713-723. DOI: 10.1007/s10584-016-1607-3.

[5] Sprague, Ralph H. (1980): A Framework for the Development of Decision Support Systems. In MIS Quarterly 4 (4), pp. 1-26. DOI: 10.2307/248957.

[6] Phillips-Wren, Gloria E.; Hahn, Eugene D.; Forgionne, Guisseppi A. (2004): A multiple-criteria framework for evaluation of decision support systems. In Omega 32 (4), pp. 323-332. DOI: 10.1016/j.omega.2004.01.003.

[7] Pareto, Vilfredo (1900): Sunto di Alcuni Capitoli di un Nuovo Trattato di Economia Pura del Prof. Pareto. In Giornale degli Economisti 20 (11), pp. 216-235.

[8] Bessette, Douglas L.; Arvai, Joseph; Campbell-Arvai, Victoria (2014): Decision support framework for developing regional energy strategies. In Environmental science \& technology 48 (3), pp. 1401-1408. DOI: 10.1021/es4036286.

[9] Czaika, Ellen; Selin, Noelle E. (2017): Model use in sustainability policy making. An experimental study. In Environmental Modelling \& Software 98, pp. 54-62. DOI: 10.1016/j.envsoft.2017.09.001.

[10] Allison, Andrew E.F.; Dickson, Mark E.; Fisher, Karen T.; Thrush, Simon F. (2018): Dilemmas of modelling and decision-making in environmental research. In Environmental Modelling \& Software 99, pp. 147-155. DOI: 10.1016/j.envsoft.2017.09.015.

[11] Schaffernicht, Martin; Groesser, Stefan N. (2011): A comprehensive method for comparing mental models of dynamic systems. In European Journal of Operational Research $210 \quad$ (1), pp. 57-67. DOI: 10.1016/j.ejor.2010.09.003.

[12] Budescu, David V.; Broomell, Stephen B.; Lempert, Robert J.; Keller, Klaus (2014): Aided and unaided decisions with imprecise probabilities in the domain of losses. In EURO J Decis Process 2 (1-2), pp. 31-62. DOI: 10.1007/s40070-013-0023-4.

[13] Alter, Steven (1977): A Taxonomy of Decision Support Systems. In Sloan Management Review 19 (1), pp. 39-57.

[14] Lurie, Nicholas H.; Mason, Charlotte H. (2007): Visual Representation. Implications for Decision Making. In Journal of Marketing 71 (1), pp. 160-177. DOI: 10.1509/jmkg.71.1.160.

[15] Sundar, S. Shyam (2004): Theorizing Interactivity's Effects. In The Information Society 20 (5), pp. 385-389. DOI: 10.1080/01972240490508072.

[16] Carpenter, S. R.; Ludwig, D.; Brock, W. A. (1999): Management of Eutrophication for Lakes Subject to Potentially Irreversible Change. In Ecological Applications
9 (3), pp. 751-771. DOI: 10.1890/10510761(1999)009[0751:MOEFLS]2.0.CO;2. 